\title{
Limpieza del vidrio por erosión iónica, previa a la deposición del recubrimiento decorativo
}

\author{
N. VERSHININ1 ${ }^{1}$ B. STRAUMAL ${ }^{1}$, C. FERRER GIMÉNEZ ${ }^{1}$, \\ A. SÁNCHEZ BOLINCHES ${ }^{2}$, A. CANTARERO ${ }^{3}$ \\ II.V.T., S.L. (Institute for Vacuum Technology), P.O. Box 47, 109180 Moscow, Russia \\ ${ }^{2}$ Departamento de Ingeniería Mecánica y de Materiales, Universidad Politécnica de Valencia, Camino de Vera, s/n, 46022 Valencia. \\ ${ }^{3}$ Instituto de Ciencia de Materiales, Universidad de Valencia, Apt. Correos 2085, 46071 Valencia.
}

\begin{abstract}
La erosión iónica es el método más eficaz para la eliminación de la capa superficial de los substratos en su fase de preparación para la deposición de recubrimientos en vacío. En el presente trabajo se describe la construcción y características de un acelerador Hall de la corriente eléctrica de abertura larga, diseñado para la limpieza, mediante erosión iónica, de superficies de substratos de vidrio, metal y plástico en forma de láminas con área extensa. Una de las ventajas del acelerador Hall es la posibilidad de uso de gases reactivos. El acelerador aquí descrito es capaz de trabajar con argón, oxígeno, nitrógeno y dióxido de carbono. Este acelerador tiene una forma de bucle muy alargado y, por tanto, dispone de una abertura larga (1400 mm en vertical). La potencia máxima del acelerador es de $10 \mathrm{~kW}$. Se presenta la característica corriente-tensión del acelerador en atmósfera de argón que permite optimizar el régimen de limpieza por erosión, encontrando el máximo de potencia para la descarga estable.
\end{abstract}

Palabras clave: acelerador Hall, erosión iónica, limpieza, vidrio.

Ionic sputtering of glass before deposition of decorative coatings

Sputter cleaning is the most reliable way for the pre-treatment of substrates before the deposition of coatings with the aid of magnetron sputtering or vacuum arc deposition. In this work, a large-aperture Hall current accelerator was developed for the sputter cleaning of large-area glasses, metals and plastics sheets. The main advantage of the Hall current accelerator is the possibility to use reactive gases. The Hall accelerator developed is able to work with argon, oxygen, nitrogen and carbon dioxide. The accelerator has the form of a very elongated loop with a large aperture (1400 $\mathrm{mm}$ in the vertical direction). The maximum power of the Hall current accelerator is $10 \mathrm{~kW}$. The current-voltage characteristic measured for argon is presented. It allows one to optimise the regime of sputter cleaning by finding the maximum power value at a stable discharge.

Keywords: Hall current accelerator, glass, sputtering, cleaning.

\section{INTRODUCCIÓN}

\subsection{Algunas dificultades de las tecnologías de recubrimien- tos sobre vidrio}

El desarrollo de las tecnologías avanzadas de recubrimientos, acompañado por la caída drástica de los costes relacionados, ha abierto las posibilidades de aplicación de estos recubrimientos fuera de las áreas tradicionales de productos de alto coste, como son las industrias de la microelectrónica, la aeronáutica o de vuelos espaciales. Hoy día estas tecnologías han llegado a penetrar en las áreas de productos de bajo coste, utilizándose en las industrias de materiales de construcción, de edificación y, en particular, de vidrio [1-4]. En los últimos años el mercado de recubrimientos sobre vidrio muestra un gran crecimiento debido, en primer lugar, a aplicaciones arquitectónicas y microelectrónicas. En estos momentos, ya es posible el diseño de recubrimientos multicapa que cumplan con todas las exigencias de una aplicación concreta con un coste aceptable. Para las industrias de edificación y automoción los avances más destacados en el tema de recubrimientos están relacionados con recubrimientos antireflectantes de máxima transmitancia de luz y de baja emisividad para el aislamiento térmico [1].
Los recubrimientos complejos multicapa, con una capa de óxido o material inorgánico conductor, permiten conseguir un vidrio con transmitancia variable de la luz [5], aumentando la aplicación de los denominados "vidrios inteligentes" ("smart windows") capaces de autoregular la intensidad de luz en una habitación.

El proceso de fabricación de estos recubrimientos, ya bastante complejo por sí mismo, se complica aun más en el caso del vidrio por la necesidad de aplicar exigencias tales como la uniformidad del espesor y la ausencia de defectos en áreas extensas, típicas de este material. Para conseguir una buena adherencia del recubrimiento, deben de eliminarse las mínimas huellas de impurezas de la superficie del vidrio. Estos requerimientos restringen fuertemente las posibilidades de selección de las técnicas adecuadas para la limpieza superficial del vidrio. Además, como las técnicas de limpieza tienen que ser de alta productividad, compatible con la velocidad de las cadenas de fabricación de vidrio, el número de estas técnicas resulta aún más reducido. Uno de los métodos de limpieza más eficaces es el de eliminación de la capa superficial por erosión. La selección acertada del método de erosión es de especial importancia en el caso de substratos de áreas extensas. 


\subsection{Comparación de dos dispositivos para la erosión superficial}

La técnica de erosión, igual que las técnicas de deposición de películas finas o de implantación de iones, utiliza un haz de iones para el tratamiento superficial [6]. A pesar de que el principio básico pueda ser el mismo para todas estas técnicas [7], cada aplicación concreta exige el diseño de una fuente de iones específica, según el rango de energías de iones y el nivel deseado de la uniformidad del haz. Para la tecnología de recubrimientos sobre el vidrio, la calidad de la limpieza de la superficie tiene una importancia crucial por su influencia en la calidad de las capas depositadas, especialmente en lo referente a la adherencia y la resistencia contra la corrosión. Hoy día está comprobado que el método de erosión de la capa superficial del substrato es el más eficaz para conseguir recubrimientos de calidad sobre vidrio, metales y plásticos. En adelante se comparan dos fuentes de haz de iones: la fuente Kaufman y el acelerador Hall de la corriente eléctrica. La calidad de limpieza superficial, que se consigue con estas fuentes es igual de alta, sin embargo, hay diferencia muy importante entre ellas desde el punto de vista de su utilización a escala industrial.

Las fuentes Kaufman son las que suelen utilizarse como fuentes de haz de iones en las instalaciones de erosión superficial [8-10]. Estas fuentes son muy atractivas por su capacidad de generar un haz de iones neutro y controlar de forma independiente la energía de los iones y la intensidad de la corriente. Además, la producción de los iones está separada del blanco y del substrato. Por sus altos niveles de control y de uniformidad las fuentes Kaufman resultan muy competitivas, comparando con las de plasma. Sin embargo, las exigencias contradictorias industriales delimitan el uso de estas fuentes [11]. Los elementos de óptica de rejilla y de cátodo son los componentes críticos de las fuentes Kaufman, que a veces exigen demasiado trabajo de mantenimiento. El cátodo, que emite los electrones para ionizar el gas de descarga, está sometido a la erosión por bombardeo con las partículas ionizadas. Según el tipo de cátodo, su tiempo de vida es del orden de las centenas de horas. El calentamiento local o presencia de gases reactivos, como el oxígeno, reducen dramáticamente el tiempo de vida del cátodo. Los componentes de óptica de rejilla, como la rejilla de pantalla y rejilla de acelerador, también resultan dañados por erosión, provocada bien por los fenómenos de carga espacial o bien por la excesiva intensidad de la corriente del haz de iones. Por estas razones, resulta necesario limitar la intensidad de la corriente del haz de iones que se extrae de la fuente. Además, estas fuentes resultan ser poco eficaces para áreas extensas, lo que es importante en la aplicación industrial de recubrimientos sobre vidrio.

Otro tipo de fuente de haz de iones es la que utiliza un acelerador Hall de la corriente eléctrica. Se ha encontrado [9] que por la forma especial de movimiento de electrones en el acelerador Hall, su banda energética de los iones del haz resulta más ancha que la de una fuente Kaufman. Las razones de este aumento de dispersión de energía la atribuyen al intercambio de las cargas y a las fluctuaciones del plasma. A pesar de esto y de que estos aceleradores son menos controlables que las fuentes Kaufman, se adecuan mejor a las exigencias de la industria [10]. La intensidad de la corriente de iones del acelerador Hall no tiene limitación por el flujo de la carga espacial. Otra ventaja de estos aceleradores consiste en la ausencia de los componentes delicados como el cátodo de la fuente Kaufman o dispositivos de óptica de rejillas. El acelerador Hall requiere un mantenimiento mínimo y la limpieza por erosión puede efectuarse con gases reactivos como oxígeno, nitrógeno o dióxido de carbono.

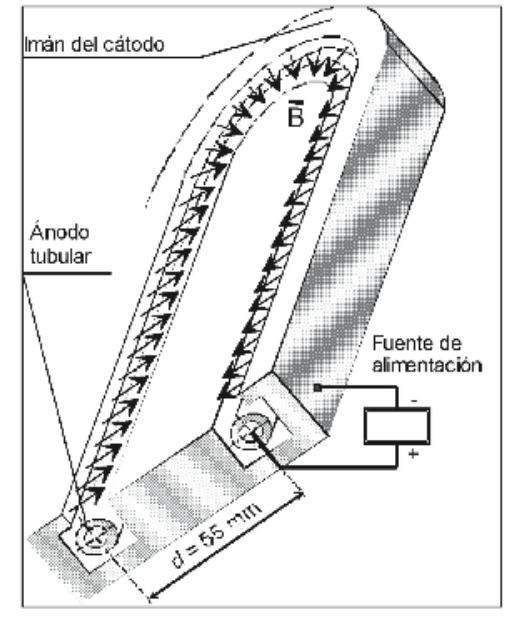

Fig. 1. Esquema del acelerador de corriente Hall, sección del plano medio. La longitud total o la abertura es de $1400 \mathrm{~mm}$.

\subsection{Acelerador Hall para el uso industrial}

En la Fig. 1 se representa un esquema del acelerador Hall. El acelerador tiene la forma de un bucle muy alargado; por esta razón la fuente dispone de dos brazos paralelos separados a distancia de $55 \mathrm{~mm}$ entre ellos y, por lo tanto, es de doble abertura. Su altura es de $1400 \mathrm{~mm}$.

El acelerador está compuesto por dos imanes permanentes yuxtapuestos, que al mismo tiempo actúan como cátodo. Dentro del canal, formado por estos dos imanes, se encuentra el ánodo tubular refrigerado con agua. Todo este dispositivo se encuentra dentro de la cámara de vacío en la que se inyecta el gas de erosión (normalmente argón). La ionización del gas y aceleración posterior de los iones, se produce por los campos magnético y eléctrico entrecruzados. El campo eléctrico surge por la diferencia entre los potenciales del ánodo y del cátodo. El campo magnético cuasi uniforme se establece entre las dos piezas polares del imán. En presencia de la atmósfera de gas a baja presión y del campo eléctrico de alta tensión se obtiene el plasma de descarga incandescente. El campo magnético atrapa los electrones del plasma y, junto con el campo eléctrico, los pone en movimiento cicloidal sobre la superficie del ánodo. Durante este movimiento, los electrones impactan con los átomos de argón y los ionizan. Los iones se mueven en trayectorias que tienen la misma forma que las de los electrones pero, como la masa de los iones es mucho más alta que la de los electrones, éstas son mucho más abiertas lo que conlleva su salida del espacio entre cátodo y ánodo a través de las aberturas, formándose el haz de iones. Este haz, al alcanzar la superficie de vidrio, la erosiona por el bombardeo y la limpia.

\section{PARTE EXPERIMENTAL}

2.1. Funcionamiento del acelerador como dispositivo de limpieza de substratos en un equipo industrial para la deposición de recubrimientos.

La abertura larga del acelerador (1400 mm en vertical) permite utilizarlo en la instalación industrial polifuncional "Nikolai", destinada a la deposición de recubrimientos sobre las superficies extensas de vidrio con la pulverización por el arco eléctrico en vacío [12-14] o con la magnetrónica. El tamaño máximo de la superficie a tratar es de 2100 × $1300 \mathrm{~mm}$. Durante el trata- 
miento las láminas pasan por delante del acelerador a una velocidad preelegida. La superficie a limpiar es perpendicular al eje del flujo de los iones. Cambiando la velocidad de desplazamiento y la potencia del acelerador se puede controlar el espesor de la capa eliminada de la superficie. El recubrimiento se deposita inmediatamente después de esta limpieza, con lo que se evita la contaminación de la superficie del substrato. La capacidad del equipo, en el caso de vidrio, es de 30 láminas por ciclo de trabajo.

\subsection{Registro de la característica corriente-tensión del acelera- dor en atmósfera de argón}

En la cámara de la instalación industrial, equipada con dos aceleradores uno enfrente del otro, se coloca una lámina de vidrio de forma descrita en el epígrafe 2.1.

A continuación, se vacia la cámara hasta una presión de 5·10 ${ }^{4} \mathrm{~Pa}$ y se inyecta el gas de trabajo, en este caso argón, hasta una presión de 0,01 Pa. Al cebar el arco, se mide la intensidad de la corriente consumida por el acelerador a distintos niveles de tensión eléctrica entre el cátodo y el ánodo.

\subsection{Registro de las curvas de erosión}

A pesar de que la industria de recubrimientos decorativos sobre vidrio es menos exigente que la de microelectrónica, es necesario conocer el grado de erosión de la superficie en función de las variables del proceso, incluido su posición respecto al flujo de iones. La distribución espacial no uniforme de la energía de los iones dentro del haz iónico provoca la no-uniformidad de la erosión de la superficie. Evaluar el efecto conjunto de no-uniformidad del haz iónico y de la posición relativa de la superficie y del haz permite la denominada "curva de erosión", que es la curva de distribución de intensidad de erosión en función de la distancia entre el punto erosionado y el plano vertical de simetría del haz de iones. Esta curva permite evaluar el espesor de la capa eliminada en el proceso industrial de limpieza de la superficie.

Para establecer la curva de erosión de la fuente se ha utilizado el siguiente método simple. Como muestras se han utilizado láminas de vidrio de $200 \mathrm{~mm}$ de ancho con marcas verticales, dibujadas en su superficie a una distancia constante entre ellas. Estas muestras se colocaron dentro de la cámara del equipo entre los dos aceleradores, destinados a limpiar las dos caras de una lámina. La posición de la muestra era extrema: a una distancia mínima del acelerador izquierdo y a una máxima, de 300 $\mathrm{mm}$, del derecho. El plano de la muestra era perpendicular al eje del haz de iones. La muestra se exponía a la acción del haz sólo por el lado marcado, utilizándose la fuente lejana, es decir, la derecha. Durante la exposición, la muestra permanecía inmóvil. Las marcas dibujadas protegían la superficie contra la erosión. Después del tratamiento, las marcas se eliminaban con alcohol, apareciendo escalones entre las partes pulverizadas y no pulverizadas. De la altura de escalones, que se medía con el perfilómetro Taylor-Hobson, se obtenían los espesores de las capas eliminadas o las velocidades de erosión. La secuencia de velocidades de erosión en función de la distancia entre el punto erosionado y el plano de simetría vertical del haz de iones, proporcionaba los puntos de la curva de erosión para la exposición utilizada.

Estos experimentos han sido realizados con vidrios silicatos y orgánicos (polimetilmetacrilato - PMMA).

\section{RESULTADOS Y DISCUSIÓN}

\subsection{Datos técnicos del acelerador}

En este trabajo se presenta el acelerador Hall de abertura larga, diseñado para la limpieza por erosión iónica de áreas extensas de substratos de vidrio, metal y plástico. Para el uso industrial la presión de argón es de $0.01 \mathrm{~Pa}$, la potencia de fuente de $6 \mathrm{kV}$ y la intensidad de la corriente de $0.5 \mathrm{~A}$. El haz de iones resultante tiene una energía media de $6 \mathrm{keV}$. La potencia máxima de la fuente es de $10 \mathrm{~kW}$.

Esto revela un avance en el desarrollo de las fuentes Hall, partiendo de la primera realización que disponía de una abertura de $10 \mathrm{~cm}$ y una energía del haz de iones de 50-75 eV [10].

\subsection{Característica corriente-tensión del acelerador Hall}

La característica corriente-tensión del acelerador con argón a presión de $2.410^{-2} \mathrm{~Pa}$ dispone de dos ramas: de "alta" y de "baja" tensión. Son bastante similares entre sí, con única diferencia en la magnitud media de tensión. Para ambas ramas la variación de tensión es relativamente lenta mientras la intensidad de la corriente varía entre unos 0,1 y 2,0 Amperios. Fuera de estos límites de intensidad de la corriente, la caída de tensión se hace muy rápida y el arco pierde la estabilidad. De estas dos ramas solo la de tensión "alta" tiene el significado industrial: a ella le corresponde la potencia de haz de iones más alta.

En la Fig. 2. está representada solo la rama de tensión "alta" de la característica. Usando esta curva, se puede optimizar el régimen de limpieza por bombardeo iónico encontrándose el máximo de potencia para la descarga estable.

\subsection{Curvas de erosión del vidrio para la fuente de haz iónico de argón}

En la Fig. 3 se representan las curvas de erosión para el vidrio silicato. Las curvas similares para el vidrio orgánico ya fueron publicadas anteriormente [15]. Tal como cabía esperar, por el comportamiento muy diferente de los vidrios orgánicos y silicatos durante el tratamiento de erosión, la forma de estas curvas depende del material de substrato.

La velocidad máxima de erosión de PMMA puede alcanzar unos $100 \mathrm{~nm} / \mathrm{min}$ [15]. Sin embargo, la resistencia térmica de los vidrios orgánicos es más baja que la de los vidrios silicatos. Por esta razón la erosión durante más de 5 min provoca el deterioro del vidrio orgánico. La superficie se cubre con una película de color marrón claro, revelando la existencia de

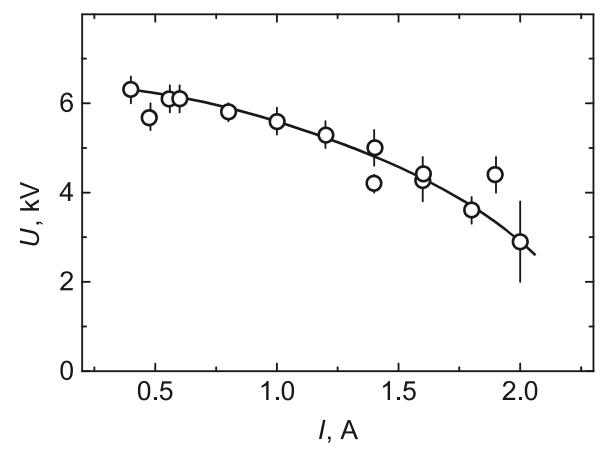

Fig. 2. Característica corriente-tensión del acelerador de corriente Hall con atmósfera de argón a presión 2.4x10-2 Pa. 
reacciones químicas. Esto significa que el proceso de limpieza del vidrio orgánico se realiza como combinación de erosión y reacciones químicas. Cabe esperar que las transformaciones de este material se producen de forma semejante a las transformaciones por radiación ionizante [16].

Las curvas de erosión de los vidrios silicatos (Fig. 3) son mucho más reproducibles en el tiempo que las de vidrios orgánicos. Otra diferencia importante entre estos casos está en que en las curvas del vidrio silicato aparece dos picos. Estos picos se corresponden con dos brazos paralelos del bucle anódico del acelerador (Fig. 1). Así que la doble abertura de la fuente se revela en las curvas para el vidrio silicato. En la región de limpieza más intensa, que es la de doble pico, la velocidad de erosión alcanza $7 \mathrm{~nm} / \mathrm{min}$. En primera aproximación la curva de erosión de la fuente puede ser considerada como un perfil de distribución de Gauss doble, con dos picos diferentes. Para una distancia de $300 \mathrm{~mm}$ de la superficie hasta la fuente, la distancia entre los picos de esta distribución Gauss resulta ser de unos $30 \mathrm{~mm}$, lo que es menor que la distancia entre las aberturas paralelas del acelerador (Fig. 1). Esto indica que el haz de la fuente es convergente y que la descripción completa del haz debería incluir la deriva de los picos en función de la distancia hasta la fuente.

\subsection{Estimación del espesor eliminado}

Durante el tratamiento industrial, el substrato de vidrio pasa por delante de la fuente con una velocidad constante, con lo que se asegura una limpieza uniforme. Esta velocidad depende de las posibilidades de la fuente y de los requisitos de la cadena de fabricación. La estimación del espesor pulverizado se puede obtener integrando la curva de erosión, expresada en unidades de velocidad de limpieza, por ejemplo en $\mathrm{nm} / \mathrm{min}$, a lo largo de anchura de la fuente. Para las velocidades propias del proceso industrial, la capa pulverizada del vidrio varía entre $0,6 \mathrm{~nm}$ para altas velocidades de alimentación y $13 \mathrm{~nm}$ para bajas velocidades. Para la velocidad más común, el espesor pulverizado es de unos $2 \mathrm{~nm}$.

\section{CONCLUSIONES}

En este trabajo se presenta la construcción de un acelerador Hall de abertura larga, diseñado y desarrollado para tratamientos de vidrio a escala industrial. La ausencia de componentes de corto tiempo de vida en su construcción hace que esta fuente resulte más adecuada para las aplicaciones industriales que una fuente Kaufman. El mantenimiento que necesita este acelerador es mínimo. Siendo utilizado para la limpieza por erosión iónica, puede trabajar bien con gases inertes bien con reactivos, como el oxígeno, nitrógeno, o dióxido de carbono. Se han determinado las curvas de erosión con argón a una distancia de $300 \mathrm{~mm}$ de la fuente para el vidrio silicato. Estas curvas han sido comparadas con las de vidrio orgánico (PMMA). En ambos casos la distribución ha resultado ser no homogénea, presentando un estrecho intervalo de distancias con una máxima intensidad de erosión. La velocidad de erosión del vidrio silicato resulta ser unas 12 veces menos que la del PMMA. El aspecto de las curvas de erosión es algo más complicado para el vidrio silicato: se resuelven dos picos que se corresponden con las dos aberturas de la fuente. El método utilizado para caracterizar la erosión permite estimar el espesor de la capa eliminada por erosión cuando la lámina del vidrio se encuentra en movimiento con una determinada velocidad respecto a la fuente. También se ha representado la característica corriente-tensión para erosión por bombardeo con iones de argón. Esta característica permite optimizar el régimen de erosión, encontrándose el máximo de potencia para la descarga estable.

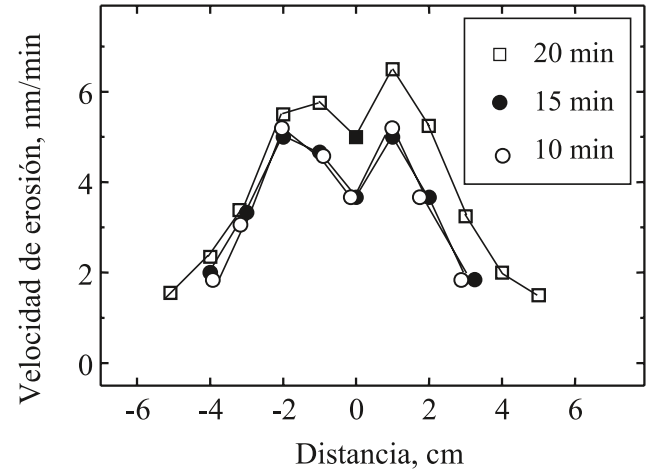

Fig. 3. Perfiles de erosión iónica del vidrio silicato para varios tiempos de pulverización. El eje horizontal es de las distancias entre los puntos erosionados de la superficie de la lámina de vidrio y el plano de simetría vertical del haz iónico.

\section{AGRADECIMIENTOS}

Este trabajo constituye una parte de trabajo general que se está desarrollando bajo la financiación del Ministerio de Ciencia y Tecnología de Rusia ("Isopress-Inter Program”), de Volkswagen Stiftung (Contrato I/74 641), de Copernicus Network (Contrato ERB IC 15 CT98 0812) y del IMPIVA (proyecto "Análisis del sector de vidrio hueco en la Comunidad Valenciana"). El inestimable apoyo de la Universidad de Valencia y de la Consellería de Cultura, Educación y Ciencia de la Generalitat Valenciana hicieron posible la estrecha colaboración entre los participantes de este grupo.

\section{BIBLIOGRAFÍA}

1. R. J. Martín Palma, J. M. Martínez Duart, A. Malats i Riera. «Recubrimientos espectralmente selectivos sobre vidrios arquitectónicos: recubrimientos de baja emisividad». Bol. Soc. Esp. Cerám. Vidrio, 37 [1], 7-12 (1998).

2. C. Zaldo. «Posibilidades del depósito mediante ablation con láser de óxidos electroópticos y ferroeléctricos». Bol. Soc. Esp. Cerám. Vidrio, 37 [2-3], 103-108 (1998).

3. J. Livage. «Optical and electrical properties of vanadium oxide films deposited from alkoxides». Bol. Soc. Esp. Cerám. Vidrio, 37 [2-3], 87-92 (1998).

4. F. Ojeda, F. J. Marti, J. M. Albella. «Preparación de recubrimientos cerámicos mediante técnicas de CVD». Bol. Soc. Esp. Cerám. Vidrio, 37 [6] 447-453 (1998).

5. B. Straumal, N. Vershinin, K. Filonov, R. Dimitriou, W. Gust. «Masked deposition of decorative coatings on large area glass and plastic sheets». Thin Solid Films 351 [1-2] 186-190 (1999)

6. D. G. Armour, P. Bailey, G. Sharples. «The use of ion-beams in thin-film deposition». Vacuum 36, 769-775 (1986)

7. P. D. Townsend, J. C. Kelly, N. E. W. Hartley, Ion implantation, sputtering and their applications, Academic Press, New York (1976)

8. H. R. Kaufman. «Technology of ion beam sources used in sputtering». Adv. Electronics \& Electron Phys. 36, 265-269. (1974).

9. H. R. Kaufman, J. M. E. Harper, J. J. Cuomo. «Developments in broad-beam ion source technology and application». J. Vac. Sci. Technol. 21, 764-767 (1982).

10. H. R. Kaufman, J. J. Cuomo, J. M. E. Harper. «Technology and application of broadbeam ion sources used in sputtering». J. Vac. Sci. Technol. 21, 725-736, (1982).

11. H. R. Kaufman. « Broad-beam ion sources: Present status and future directions». J. Vac. Sci. Technol. A 4 [3], 764-771 (1986).

12. N. Vershinin, B. Straumal, W. Gust. «Vacuum arc deposition of Mo films». J. Vac. Sci. Technol. A 14 [6], 3252-3255 (1996).

13. N. F. Vershinin, V. G. Glebovsky, B. B. Straumal, W. Gust, H. Brongersma. «Vacuum arc deposition as a complementary technology to laser processing». Appl. Surf. Sci., 109/110, 437-441 (1996).

14. B. B. Straumal, W. Gust, N. F. Vershinin, M. Friesel, M. Willander. «Vacuum arc deposition of Ni-Ti gradient coatings». Surf. Coat. Techn. 100-101 [1-3], 316-319 (1998).

15. N. Vershinin, B. Straumal, K. Filonov, R. Dimitriou, W. Gust, M. Benmalek. «Hall current accelerator for the pre-treatment of large area glass sheets». Thin Solid Films 351 [1-2] 172-175 (1999).

16. D. Evans, M. A. Crook. «Irradiation of plastics: damage and gas evolution». MRS Bulletin 22, 36-40 (1997).

Recibido: $18-9-99$

Aceptado: 3-5-00 